\title{
What can We Learn About the Return Migration of Fraser River Sockeye Salmon from Catches in Alaska?
}

\author{
Pasan Samarasin$^{1}$, Stephen J. Latham ${ }^{1}$, Charles M. Guthrie III², and H. Andres Araujo ${ }^{3}$ \\ ${ }^{1}$ Pacific Salmon Commission, Vancouver, British Columbia, Canada
}

\author{
${ }^{2}$ National Oceanographic and Atmospheric Administration, National Marine Fisheries Service, Alaska Fisheries \\ Science Center, Auke Bay Laboratories, Juneau, Alaska, USA
}

${ }^{3}$ Fisheries and Oceans Canada, Pacific Biological Station, Nanaimo, Canada

Keywords: migration route, marine distribution, Fraser River sockeye

At large spatial scales, aggregating across populations, North American sockeye salmon populations have largely overlapped marine distributions in the North Pacific Ocean (e.g., Bristol Bay and British Columbia stocks, Quinn 2018), but we do not have a good understanding of the marine distribution of sockeye salmon at smaller scales. For example, we do not know how sockeye salmon from different British Columbian rivers are distributed in the North Pacific Ocean. Similarly, we do not know where genetically distinct stocks within a river are distributed in the ocean. Blackbourn (1987) hypothesized that different Fraser River sockeye stocks may reside in different areas in the North Pacific Ocean based on correlations of their run timings and sea surface temperatures in different North Pacific 'grids', but this remains conjectural and based on only circumstantial evidence. Understanding the marine distributions and migratory routes at more granular levels (e.g., stocks within rivers) is beneficial because conservation and fisheries management mostly occur at such scales. Also, it is of considerable scientific interest to understand mechanisms governing long-distance animal movement. Here, we focus on the marine distributions and migratory paths of Fraser River sockeye salmon stocks, whose fisheries management is governed by the Pacific Salmon Treaty between the United States and Canada.

In their return migration from the open ocean, adult Fraser sockeye are thought to take a northeastward route from marine feeding grounds, and then migrate southeast as they approach coastal waters toward southeast Alaska to the Fraser River (McKinnell et al. 2012). Historically, Fraser River stocks have a highly consistent sequence of arrival at the river mouth (Woodey 1987). The earliest stocks arrive at the river mouth in late June and the last stocks arrive at the river mouth in late August. This pattern is well established with historical data and any useful migration model must be consistent with the sequence of stock-specific return. Here, we evaluate how well empirical data match results predicted by two simple return migration models.

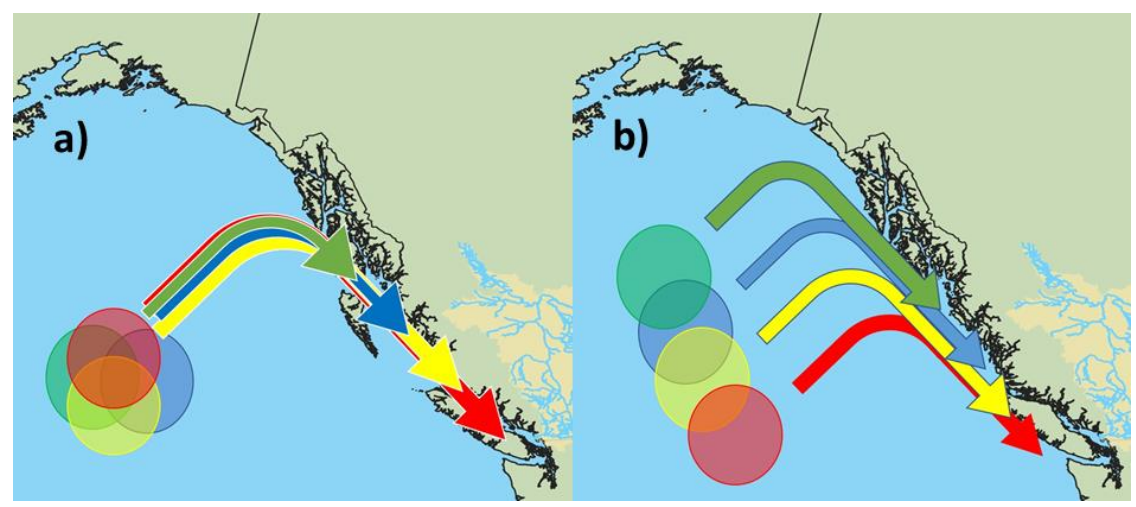

Fig. 1. Two migration models evaluated in this study: a) model 1Fraser stocks with highly overlapped marine distributions and very similar migration routes (initiation date of the return migration differs among stocks and accounts for different arrival dates), b) model 2-Fraser stocks with more distinct marine distributions but similar migration trajectories (timing need not differ among stocks to account for different dates of arrival at the Fraser River).

The first model hypothesizes that different Fraser River stocks have similar or highly overlapping distributions in the North Pacific Ocean shortly before the start of their return migration (Fig. 1a). The returning adults swim at similar speeds along very similar migratory paths all the way to the Fraser River, but they start their migrations at different times, thereby accounting for their different arrival timing. Thus, different stocks would be sequentially available to various coastal fisheries during different weeks as they migrate toward the mouth of Fraser River. According to this model, there would be no difference in stock-specific availability to coastal fisheries at an annual scale because all stocks eventually migrate through the same areas. The second model hypothesizes that different Fraser stocks have less overlapped spatial distributions shortly before the start of their return migration but share a similar trajectory in returning to the river mouth (Fig. 1b). Because their initial distributions differ, the paths of their

All correspondence should be addressed to P. Samarasin. 
return migrations are also spatially offset. This accounts for their different return timing and results in some stocks having a higher latitude of landfall than others, resulting in differential stock-specific availability for coastal fisheries.

Fraser River sockeye salmon caught in fisheries in Southeast Alaska (henceforth "SEAK"), North of Vancouver Island (NVI) and South of Vancouver Island (SVI) were analyzed (Fig. 2). Genetic stock identification was used to identify the origin of fish caught in these fisheries and only fish identified as being from the Fraser River with a probability greater than 50\% were further examined. Table 1 provides sample sizes of the Fraser River sockeye caught in the three fisheries from 2005-2017. The program CBayes (Neaves et al. 2005) was used to estimate probabilities of stock origin for each individual. At an annual scale, we compared the relative proportions of each stock detected at the three fishing locations. Although NVI and SVI catches spanned the whole migration season, SEAK catches were possibly missing very early timed stocks because of timing of the fishery. Therefore, we also compared the stock compositions of weekly catches among the three locations by employing a timing index. The purpose of this analysis was two-fold: 1 ) to determine if differences in the annual stock-specific results were driven by differences in fisheries timing; and 2) to evaluate if timing compositions of catches from equivalent dates differ among locations and if observed patterns are consistent with model expectations. The timing index integrated the historical run-timing data of each Fraser stock to calculate a weighted average timing value for the weekly catch, allowing comparison of the average timing of the catch for any given week among fisheries locations. The index has a relatively low value when the catch is composed of mostly early-arriving stocks and a relatively high value when the catch is composed of late-arriving stocks. We compared the rate of change of the timing index over weeks for the three locations using linear regression analyses.

Table 1. Sample sizes of Fraser River sockeye salmon (probability > 0.5) caught in Southeast Alaska (SEAK), North of Vancouver Island (NVI), and South of Vancouver Island (SVI).

\begin{tabular}{cccc}
\hline Year & SEAK & NVI & SVI \\
\hline 2005 & 236 & 1662 & 1760 \\
2006 & 25 & 3430 & 2947 \\
2007 & 225 & 2321 & 3143 \\
2009 & 52 & 2646 & 2759 \\
2011 & 74 & 2792 & 2443 \\
2012 & 127 & 1782 & 2814 \\
2013 & 432 & 2210 & 2675 \\
2014 & 352 & 3726 & 1666 \\
2015 & 220 & 2072 & 1225 \\
2016 & 135 & 1791 & 1572 \\
2017 & 299 & 2792 & 2128 \\
\hline Total & 2185 & 27224 & 25132 \\
\hline
\end{tabular}

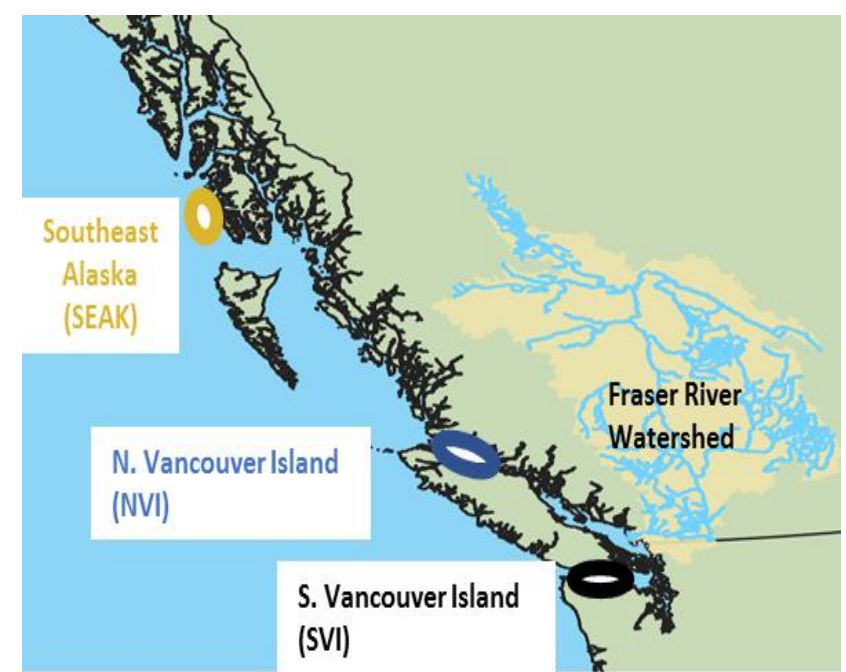

Fig. 2: Fraser sockeye sampling locations in this study (SEAK fishery locations are approximately $700 \mathrm{~km}$ northwest of NVI).

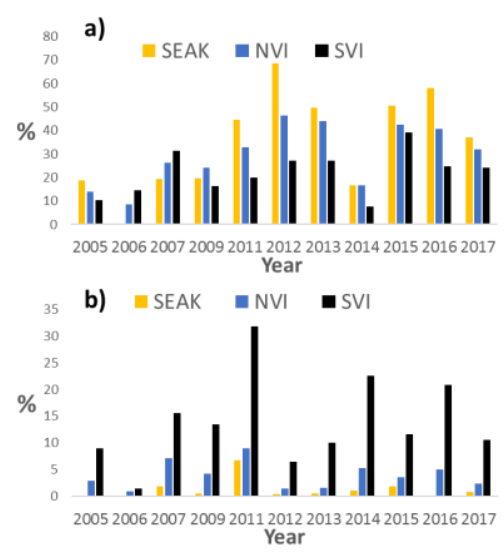

Fig. 3. Annual prevalence of Chilko and Harrison sockeye in catches from SEAK, NVI and SVI: a) Chilko sockeye, b) Harrison sockeye. 
In most years, Chilko sockeye was the most abundant stock of Fraser sockeye salmon, and its annual proportions showed a north-south latitudinal trend among the three fisheries. The Chilko proportion was highest in SEAK catches, intermediate in NVI catches, and lowest in SVI catches (Fig. 3a). This trend was observed consistently since 2011. For the Harrison stock, which exhibits a sea-type life history where juveniles migrate to the oceans in their first year, the opposite latitudinal trend was observed. The proportion of Harrison was highest in SVI catches, intermediate in NVI catches, and lowest in SEAK catches (Fig. 3b). This trend was observed in all years with available data (2005-2007, 2009, 2011-2017).

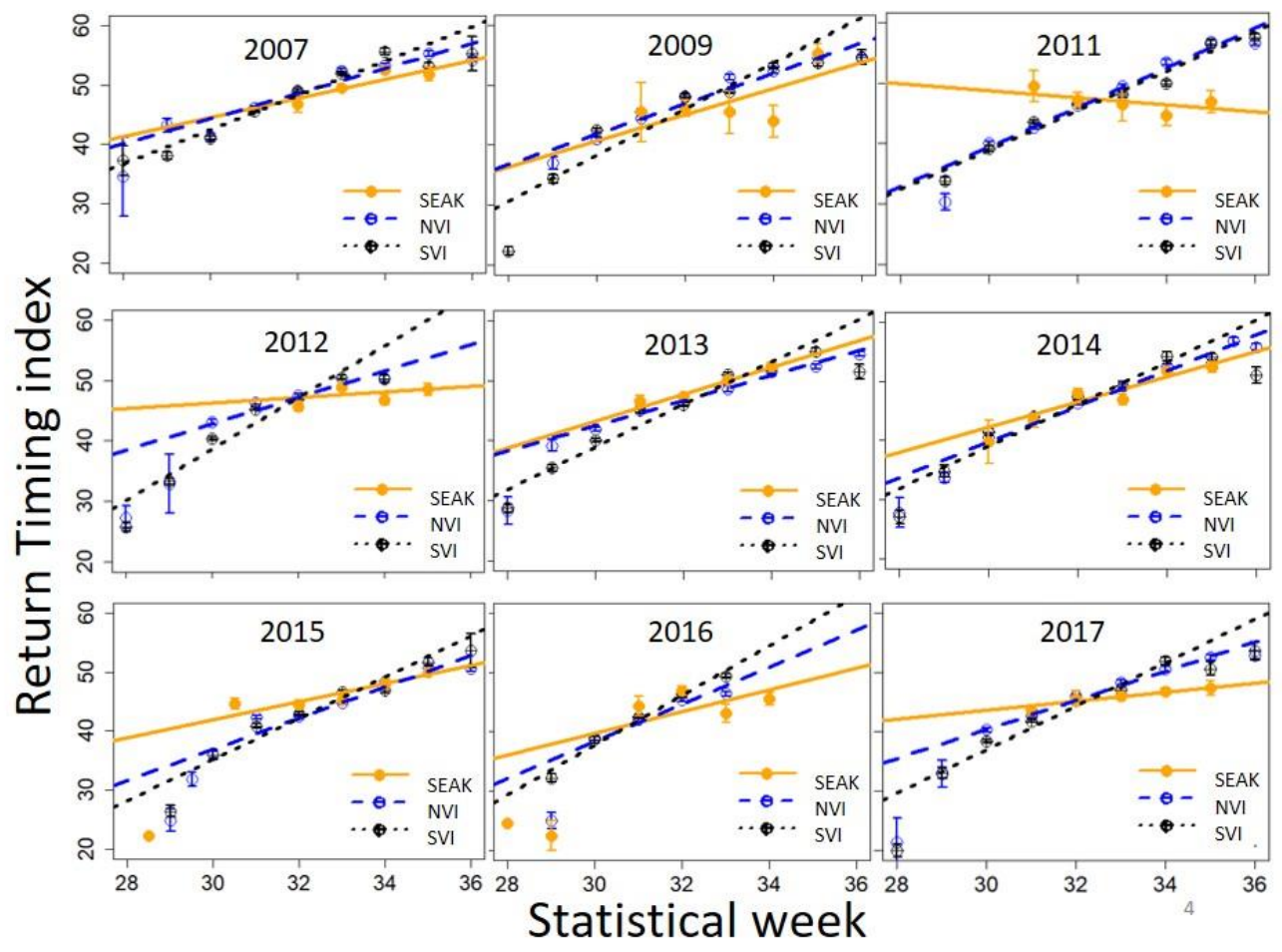

Fig. 4. Linear regression of the timing indices (weighted averages of stock-specific arrival timing) of weekly catches for SEAK, NVI, and SVI from 2007-2017.

The timing index analysis showed consistent trends among years, with the same relative positions and slopes from 2007-2017, with only 2011 being exceptional (Fig. 4). Typically, the slope of the timing index trend line was steepest for SVI, intermediate for NVI, and shallowest for SEAK. The y-intercept was lowest for SVI, intermediate for NVI, and highest for SEAK. Because of differences in slopes and intercepts, the timing index trend lines of the three locations typically intersected near or during statistical week 32 , which is near the peak of the migration season with respect to timing near the mouth of the Fraser River.

Our results suggest that Fraser stocks differ in their availability to various coastal fisheries either due to differences among stocks in their marine distributions at the start of the return migration or because of the migration route itself. For example, Harrison stock proportions among the three areas indicate Harrison fish rear in a more southerly location of the North Pacific than Chilko sockeye, or that they take a more southerly route, or both. The annual stock proportion results seem more consistent with the second rather than the first migration model because the differences in stock proportions among the three fishery locations could be accounted for by initial offsets in latitude. However, observed trends at the annual level could result from differences in fisheries timing as well. Whereas annual samples from NVI and SVI span the whole sockeye migration season and are weighted toward abundance of Fraser stocks, SEAK fisheries target pink salmon and Fraser sockeye are bycatch (i.e., the distribution of catch across weeks may not reflect the abundance of Fraser sockeye). Examination of the timing index allowed us to account for this potential problem.

Both hypothesized migration models predict parallel timing index trend lines for the three locations. Furthermore, because we expected Fraser sockeye to migrate in a southeastward direction as they approach coastal 
waters, we predicted the SEAK trend line to be highest (i.e., with the latest mean timing index for any given date) and SVI to be lowest. However, observed results were not consistent with these expectations. In all years (20072017), the three regression lines intersected, which clearly disproves the first migration model. These results are also not consistent with the second migration model because later in the sockeye salmon migration season, SEAK timing indices were lower than both NVI and SVI. Sockeye stocks migrating along similar trajectories to the Fraser River from even very different starting spatial distributions cannot produce such results while also faithfully reproducing the sequential arrival of stocks. Overall, our results suggest that the returning Fraser sockeye stocks have different starting spatial distributions and take different paths when returning to the Fraser River, requiring a more complex migration model to explain consistently observed trends.

Acknowledgements - This analysis was made possible by the sampling and other efforts of the Alaska Department of Fish and Game and by cooperation among ADF\&G, NOAA, DFO, and the PSC.

\section{REFERENCES}

Blackbourn, D.J. 1987. Sea surface temperature and pre-season prediction of return timing in Fraser River sockeye salmon (Oncorhynchus nerka). In Sockeye salmon (Oncorhynchus nerka) population biology and future management. Edited by H.D. Smith, L. Margolis, and C.C Wood. Can. Spec. Publ. Fish. Aquat. Sci. 96: 296306.

McKinnell, S.M., E. Curchitser, C. Groot, M. Kaeriyama, and K.W. Myers. 2012. PICES advisory report on the decline of Fraser River sockeye salmon Oncorhynchus nerka (Steller 1743) in relation to marine ecology. PICES Sci. Rep. No. 41. 149 pp.

Neaves, P.I., C.G. Wallace, J.R. Candy, and T.D. Beacham. 2005. CBayes: computer program for mixed stock analysis of allelic data, version v6.01. (Available at http://www.pac.dfo-mpo.gc.ca/sci/mgl/Cbayes_e.htm)

Quinn, T.P. 2018. The behavior and ecology of Pacific salmon and trout: second edition. Washington Univ. Press, Seattle.

Woodey, J.C. 1987. In-season management of Fraser River sockeye salmon (Oncorhynchus nerka): meeting multiple objectives. In Sockeye salmon (Oncorhynchus nerka) population biology and future management. Edited by H.D. Smith, L. Margolis, and C.C Wood. Can. Spec. Publ. Fish. Aquat. Sci. 96: 367-374. 